\title{
MRI Measurement of Placental Perfusion and Fetal Blood Oxygen Saturation in Normal Pregnancy and Placental Insufficiency
}

\author{
Rosalind Aughwane $^{1,2}$, Magdalena Sokolska $^{3}$, Alan Bainbridge ${ }^{3}$, David \\ Atkinson $^{4}$, Giles Kendall ${ }^{2}$, Jan Deprest ${ }^{5}$, Tom Vercauteren ${ }^{1,6}$, Anna David ${ }^{2}$, \\ Sebastien Ourselin ${ }^{1,6}$, and Andrew Melbourne ${ }^{1,6}$ \\ ${ }^{1}$ Dept. Medical Physics and Biomedical Engineering, University College London, UK. \\ ${ }^{2}$ Institute for Women's Health, University College Hospital, London, UK . ${ }^{3}$ Medical \\ Physics, UCH, London, UK. ${ }^{4}$ Centre for Medical Imaging, UCL, UK. ${ }^{5}$ University \\ Hospital KU Leuven, Belgium. ${ }^{6}$ BMEIS, Kings College London, UK.
}

\begin{abstract}
The placenta is essential for successful pregnancy outcome. Inadequate placenta development leads to poor placental perfusion and placental insufficiency, responsible for one third of antenatal stillbirths. Current imaging modalities provide poor clinical assessment of placental perfusion and pregnancy outcome. In this work we propose a technique to estimate the vascular properties of retro-placenta myometrial and placental perfusion. The fetal blood saturation is a relative unknown, thus we describe a method to simultaneously estimate the fetal blood volume in addition to the fetal blood $\mathrm{T} 2$ relaxation time from which we can estimate this parameter. This information may prove useful for predicting if and when a placenta will fail, and thus when a small baby must be delivered to have the best neurological outcome. We report differences in vascular compartments and saturation values observed between 5 normal pregnancies, and two complicated by placental insufficiency.
\end{abstract}

\section{Introduction}

The placenta is a unique organ, being perfused simultaneously by two or more individuals. Assessing placental perfusion is key to understanding and diagnosing placental insufficiency (PI), which is a significant cause of morbidity and mortality, accounting for one third of antenatal, and one quarter of intrapartum stillbirths in high income countries [1]. PI occurs when the maternal spiral arteries fail to remodel normally in early pregnancy. This leads to inadequate maternal perfusion of the placenta, the fetus becomes hypoxic affecting cognitive development and if not delivered prematurely may ultimately die.

Evaluating placental function using magnetic resonance imaging (MRI) is a growing research area [2-4]. Several MRI modalities have been investigated to monitor placental blood flow and function, each with their own advantages and disadvantages [4]. Diffusion weighted imaging (DWI) is becoming increasingly widespread in abdominal and placental imaging. When combined with the intravoxel incoherent motion model (IVIM[5]) of blood flow in capillaries, it provides 
a non-invasive method of measuring tissue properties relating to flow and perfusion. T2 relaxometry, made possible by the acquisition of images with variable echo-time, provides additional information on the static tissue composition, and $\mathrm{T} 2$ relaxation times have been shown to be significantly shorter in placenta insufficiency [3]. Both techniques have been proposed for placental imaging $[2,3]$ but how best to measure the microstructural and microvascular properties of placental tissue remains an open question. The retro-placental myometrium is the closest tissue to the placenta from which to estimate maternal perfusion and blood relaxation time without the influence of signal from fetal perfusion, which may also give interesting information on the pathophysiology of PI. We also explore the theory that fitting fetal blood T2 will allow us to estimate fetal blood saturation, and that this may be a valuable measurement of placental function.

\section{Methods}

Data The study was approved by the local research ethics committee and all participants gave written informed consent. Five women in mid-pregnancy (between $28+4$ to $34+0$ gestational weeks) with normal pregnancies, and two complicated by placental insufficiency $(25+0$ and $27+2$ gestational weeks) were included. Obstetric ultrasound confirmed normal fetal growth ( $>10$ th centile) and Dopplers in the control group. Ultrasound in the PI group showed estimated fetal weight $<1$ st centile, and abnormal fetal umbilical artery Doppler.

Imaging was performed on 1.5T Siemens Avanto, at 7 b-values (b) $(0,50,100$, 150, 200, 400, 600s.mm $\left.\mathrm{mm}^{-2}\right)$ and ten echo times (t) $(81,90,96,120,150,180,210$, $240,270,300 \mathrm{~ms})$. All echo times were acquired at b-value 0 , to allow T2 fitting, and all b-values at $\mathbf{t}=96 \mathrm{~ms}$. In addition, data was acquired at b-value 50, 200 for $\mathbf{t}=(81,90,120,150,180,210,240) \mathrm{ms}$. Voxel resolution was $1.9 \times 1.9 \times 6 \mathrm{~mm}$. To minimise the effect of motion we first used an open-source non-rigid registration routine to align all images. Masks were then drawn manually within the tissue bounadry for the placenta and retro-placental myometrium in multiple slices.

DECIDE - multi-compartment placenta modelling The Diffusion-rElaxation Combined Imaging for Detailed Placental Evaluation (DECIDE) model is a multi-compartment model of placental perfusion that combines T2 relaxometry and diffusion weighted imaging [6]. Intercapillary fetal blood has high pseudodiffusivity, $d^{*}$, and long T2 relaxation time, $T_{2}^{f b}=1 / r_{2}^{f b}$ and volume fraction $f$. Maternal blood with volume fraction $\nu$, is in the intervillous space, as opposed to intravascular, and therefore has lower diffusivity $d$, and slow relaxation $r_{2}^{m b}$. Finally, the remaining signal from the tissue has low diffusivity $d$, and rapid relaxation, $r_{2}^{t}$, associated with dense tissue.

Extending the DECIDE model to fit T2 relaxation times Estimating T2 relaxation values from the literature as in [6] may add bias to the model, as both maternal and fetal blood characteristics are different from the normal, healthy 
adult. It is normal for pregnant women to have a physiological anaemia, and therefore reduced haematocrit. This may affect the T2 relaxation time of blood, which is known to be sensitive to haematocrit and oxygen saturation [7]. We adapt the DECIDE model [6] to compare typical T2 values of the maternal and fetal blood [7]. Fetal blood has higher hematocrit and lower oxygen saturation than adult blood. Deoxygenated fetal blood in the umbilical artery (supplying the placenta) at 30 weeks gestational age is estimated to be $65 \%$ saturated, and oxygenated blood returning to the fetus in the umbilical vein is estimated to be $85 \%$ saturated [8]. This is much lower than adult saturations of $97-100 \%$.

Model-fitting Routine We develop a bespoke fitting routine for this data to improve model-fitting performance with two key features:

ROI parameter initialisation In the presense of noisy data, non-linear models with several free parameters can be prone to fitting to local minima. We avoid this situation by making use of model-fitting results obtained from average ROI signal curves. This has the effect of boosting the signal-to-noise ratio, yielding robust ROI parameter estimates from the average signal curve which make reasonable starting estimates for fitting at the voxel-level within the ROI. We thus initialise our non-linear fitting routines with parameter estimates from larger placental and myometrial regions of interest.

Fitting of independent parameters Models often contain parameters which are dependent only weakly on some parts of the underlying data. A good example of this is in diffusion MRI where ADC measurements can be robustly obtained from high b-value mono-exponential data entirely separate from low b-value perfusion effects. This technique is a common approach for IVIM model-fitting and we make use of this constraint when fitting our data. We also make use of this technique when applying the standard IVIM model to both the myometrial and placenta data sets.

Myometrium Model-fitting The myometrium is maternal-perfused, highly-vascular, muscular tissue and is not expected to have a significant pooled-fluid compartment. Thus it is reasonable to assume that there are two-compartments representing an IVIM-like blood pool at high oxygen saturation and a dense tissue space of much lower T2 (Equation 1).

$$
S(b, t)=S_{0}\left[f e^{-\mathbf{b} d^{*}-\mathbf{t} r_{2}^{m b}}+(1-f) e^{-\mathbf{b} d-\mathbf{t} r_{2}^{t}}\right]
$$

1. ADC d fitting We apply log-linear fitting of both whole-ROI and voxel-wise $d$ values to the data with b-value $b>100$ for fixed echo-time.

2. Estimation of myometrial maternal blood T2 Equation 1 is fitted to the high SNR average signal curve from the whole myometrial ROI. Average wholeplacenta estimates of $f, d^{*}, r_{2}^{m b}$ and $r_{2}^{t}$ are obtained. ADC, $d$ is constrained as in step 1.

3. Estimation of myometrial volume fractions For each voxel we fix the local value of the ADC (step 1) and obtain non-linear fits of $f, d^{*}, r_{2}^{m b}$ and $r_{2}^{t}$, initialised with the global tissue estimates from step 2. 
Placenta Model-fitting We apply the DECIDE model to fit placental tissue (Eq. 2) with variables as defined above.

$$
S(b, t)=S_{0}\left[f e^{-\mathbf{b} d^{*}-\mathbf{t} r_{2}^{f b}}+(1-f) e^{-\mathbf{b} d}\left(\nu e^{-\mathbf{t} r_{2}^{m b}}+(1-\nu) e^{-\mathbf{t} r_{2}^{t}}\right)\right]
$$

We apply the same fitting approach described as for the myometrium, modiying step 2 accordingly so that we may fit fetal blood relaxation $r_{2}^{f b} \cdot r_{2}^{m b}$ and $r_{2}^{t}$ are held fixed at literature values of $(240 \mathrm{~ms})^{-1}$ and $(46 \mathrm{~ms})^{-1}$ respectively, whilst all other parameters are fitted.

Estimation of in utero fetal blood oxygen saturation Fitting an emperical curve to the data in [7] of the form $a /\left(1+e^{-b(s-c)}\right)$ enables us to estimate oxygen saturation values for known T2. Fitted parameters for this curve, given fractional saturation $s$, are $\mathrm{a}=386 \mathrm{~ms}, \mathrm{~b}=0.36, \mathrm{c}=0.88$. Thus we are able to find approximate saturation values for each known T2 blood pool (Figure 5).

\section{Results}

DECIDE fitting of retro-placental myometrium and placenta One case was excluded from the control dataset due to motion artefact as the myometrium is very thin and therefore sensitive to motion. Figure 2 shows histograms of the voxel-by-voxel fit for the IVIM and DECIDE models of the myometrium in the four included control cases. Table 1 shows the mean and standard deviation for $f, d^{*}, d$, T2 maternal blood and T2 myometrium using the IVIM and DECIDE fit. These values are plausible given that myometrium is very vascular in normal pregnancy [9]. Mean T2 of maternal blood was $202.17 \pm 92.98 m s$, and mean T2 of myometrium $123.63 \pm 6.71 \mathrm{~ms}$. Given that T2 blood was in keeping with the literature value of $240 \mathrm{~ms}$, the maternal blood T2 was fixed at $240 \mathrm{~ms}$ for all subsequent placental DECIDE fits.

Figure 3 shows histograms of the voxel-by-voxel fit for the placenta for the five control cases. Table 1 shows the mean ( \pm standard deviation) for $f, d^{*}, d$, and T2 fetal blood using the IVIM and DECIDE model fit. Mean T2 of fetal blood was $164.86 \pm 26.66 \mathrm{~ms}$.

Comparing Control and Placental Insufficiency Myometrium and Placenta Figure 4 shows histograms for the voxel-wise fit of all control and PI data for the myometrium and the placenta. Table 1 presents control and PI mean parameter estimates of $f, d^{*}, d, \nu$, and T2 relaxation times. Both the IVIM and DECIDE mean $f$ for myometrium, representing maternal perfusion, is lower in PI compared to control cases. Placental $d$ was lower in the PI compared to control placenta with both models. Placental IVIM $f$ was lower in PI compared to control, whereas the DECIDE $f$ was higher in PI compared to control . However, in the DECIDE fit $\nu$ was lower in PI compared to control, and the T2 of fetal blood was also lower in PI. 


\begin{tabular}{|c|c|c|c|c|c|c|c|c|c|}
\hline \multirow[b]{3}{*}{ Parameter } & \multicolumn{4}{|c|}{ Myometrium } & \multicolumn{5}{|c|}{ Placenta } \\
\hline & \multicolumn{2}{|c|}{$\begin{array}{c}\text { IVIM Fit } \\
(\text { mean }( \pm \text { SD }))\end{array}$} & \multicolumn{2}{|c|}{$\begin{array}{c}\text { T2 IVIM Fit } \\
\text { (mean }( \pm \text { SD }))\end{array}$} & \multirow[b]{2}{*}{ Parameter } & \multicolumn{2}{|c|}{$\begin{array}{c}\text { IVIM Fit } \\
(\text { mean }( \pm \text { SD }))\end{array}$} & \multicolumn{2}{|c|}{$\begin{array}{c}\text { DECIDE Fit } \\
(\text { mean }( \pm \text { SD }))\end{array}$} \\
\hline & Control & $\mathrm{PI}$ & Control & $\mathrm{PI}$ & & Control & $\mathrm{PI}$ & Control & $\mathrm{PI}$ \\
\hline$f$ & $\begin{array}{c}0.480 \\
(0.087) \\
\end{array}$ & $\begin{array}{c}0.285 \\
(0.176) \\
\end{array}$ & $\begin{array}{c}0.443 \\
(0.008) \\
\end{array}$ & $\begin{array}{c}0.149 \\
(0.031) \\
\end{array}$ & $f$ & $\begin{array}{c}0.260 \\
(0.019) \\
\end{array}$ & $\begin{array}{c}0.240 \\
(0.024) \\
\end{array}$ & $\begin{array}{c}0.218 \\
(0.028) \\
\end{array}$ & $\begin{array}{c}0.293 \\
(0.083) \\
\end{array}$ \\
\hline $\begin{array}{c}\mathrm{d}^{*} \\
\left(\mathrm{~mm}^{2} \mathrm{~s}^{-1}\right) \\
\end{array}$ & $\begin{array}{c}0.051 \\
(0.013) \\
\end{array}$ & $\begin{array}{c}0.048 \\
(0.018) \\
\end{array}$ & $\begin{array}{c}0.044 \\
(0.008) \\
\end{array}$ & $\begin{array}{c}0.037 \\
(0.008) \\
\end{array}$ & $\begin{array}{c}\mathrm{d}^{*} \\
\left(\mathrm{~mm}^{2} \mathrm{~s}^{-1}\right)\end{array}$ & $\begin{array}{c}0.034 \\
(0.003) \\
\end{array}$ & $\begin{array}{c}0.044 \\
(0.008) \\
\end{array}$ & $\begin{array}{c}0.073 \\
(0.097) \\
\end{array}$ & $\begin{array}{c}0.060 \\
(0.022) \\
\end{array}$ \\
\hline $\begin{array}{c}\mathrm{d} \\
\left(\mathrm{mm}^{2} \mathrm{~s}^{-1}\right)\end{array}$ & \begin{tabular}{c|}
0.0016 \\
$(0.0003)$ \\
\end{tabular} & $\begin{array}{c}0.0017 \\
(0.0001) \\
\end{array}$ & $\begin{array}{c}0.0016 \\
(0.0003) \\
\end{array}$ & $\begin{array}{c}0.0017 \\
(0.0001) \\
\end{array}$ & $\begin{array}{c}\mathrm{d} \\
\left(\mathrm{mm}^{2} \mathrm{~s}^{-1}\right)\end{array}$ & $\begin{array}{c}0.0017 \\
(0.0001) \\
\end{array}$ & $\begin{array}{c}0.0014 \\
(0.0001) \\
\end{array}$ & $\begin{array}{c}0.0017 \\
(0.0001) \\
\end{array}$ & $\begin{array}{c}0.0014 \\
(0.0001) \\
\end{array}$ \\
\hline T2 Blood (ms) & & & $\begin{array}{l}202.17 \\
(92.98) \\
\end{array}$ & $\begin{array}{l}243.78 \\
(74.32) \\
\end{array}$ & v & & & $\begin{array}{c}0.314 \\
(0.068) \\
\end{array}$ & $\begin{array}{c}0.288 \\
(0.028) \\
\end{array}$ \\
\hline T2 Myometrium (ms) & & & \begin{tabular}{|l|}
123.63 \\
$(6.71)$ \\
\end{tabular} & $\begin{array}{c}87.93 \\
(24.24) \\
\end{array}$ & \begin{tabular}{|c|}
$\begin{array}{c}\text { T2 Fetal Blood } \\
(\mathrm{ms})\end{array}$ \\
\end{tabular} & & & $\begin{array}{l}164.86 \\
(26.66)\end{array}$ & $\begin{array}{l}136.90 \\
(42.05) \\
\end{array}$ \\
\hline
\end{tabular}

Tab. 1. Mean ( \pm standard deviation) values for the voxel wise fit of all included datasets $(n=4$ control, $n=2$ PI) for myometrial and placental IVIM and DECIDE.

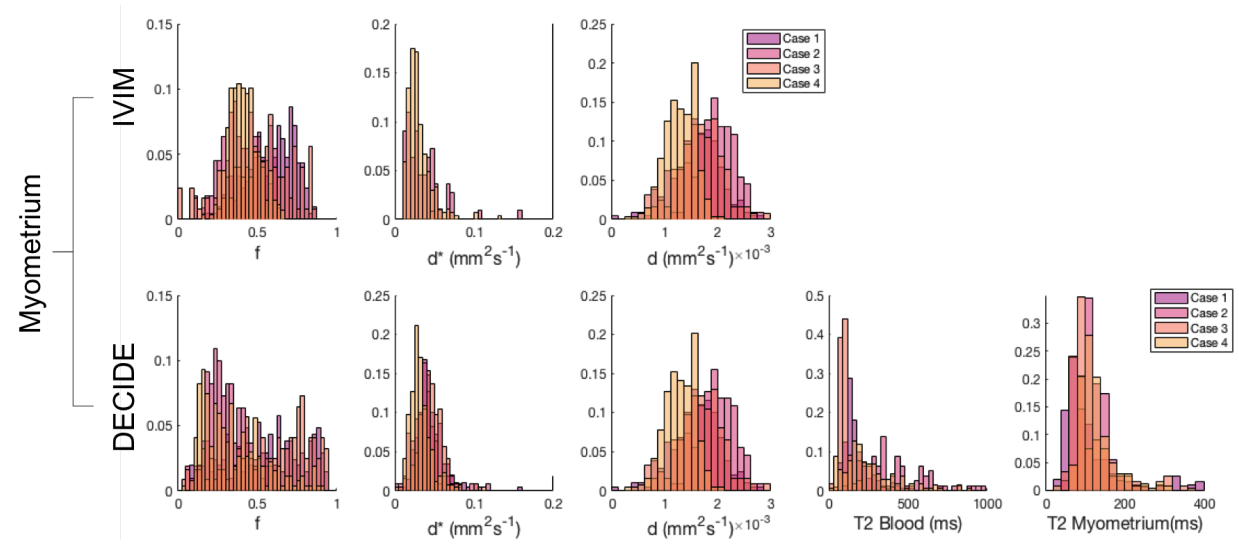

Fig. 2. Voxel-wise parameter histograms of maternal blood and myometrium.

Estimating fetal blood saturation Figure 5 shows the combined control and PI histograms for estimated fetal blood saturation, based on voxel-wise fetal blood T2 fit. Mean control fetal blood saturation was $69.14 \pm 20.12 \%$, whereas mean PI fetal blood saturation was $46.36 \pm 31.71 \%$.

\section{Discussion}

This work investigates MR imaging of placental perfusion, and the potential to estimate fetal blood saturation non-invasively for the first time. The model divides the placental signal into three compartments, relating to maternal and fetal perfusion of the placenta and the placental tissue. This work extends this model by fitting $\mathrm{T} 2$ relaxation values for fetal and maternal blood alongside data 


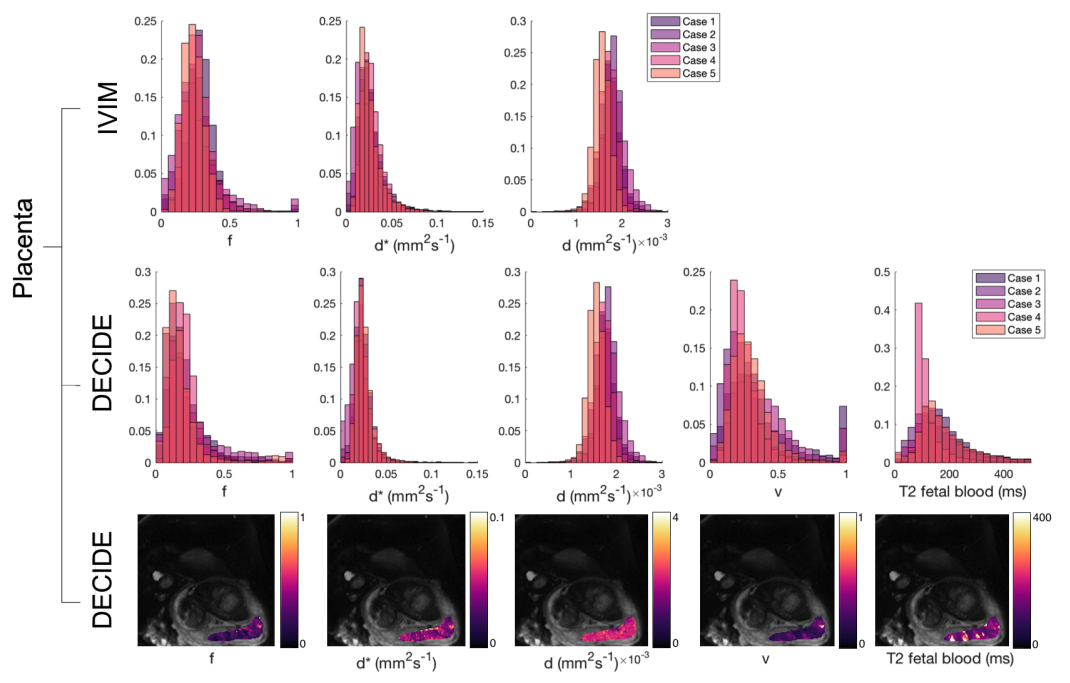

Fig. 3. Voxel-wise placenta parameter histograms for the five control dataset, showing $f, d^{*}, d, \nu$ and T2 of fetal blood. Row one; IVIM model, Row two; DECIDE model, with free fitting for fetal blood T2.

from [7] to estimate blood saturation showing differences between pregnancies complicated by PI and controls.

Maternal blood T2 relaxation time was investigated within the retro-placental myometrium. This was chosen as it is a simplified model, only needing to fit two compartments. In addition, this is likely to be a relevant tissue in investigating placental function, as differences in spiral artery perfusion are known to be an important factor in developing PI. Both the IVIM and DECIDE model gave high value for $f$ and $d^{*}$, which is feasible given that the myometrium is highly vascular in normal pregnancy, providing low resistant, high volume perfusion to the placenta. The DECIDE model found a mean maternal blood T2 relaxation time of $(202.17 \pm 92.98 \mathrm{~ms})$, and a mean tissue T2 relaxation time of $123.63 \pm 6.71 \mathrm{~ms}$. These relaxation times are consistent with those in the literature [9].

In the placenta, the DECIDE model gave a lower $f$ value than the IVIM model (0.218 DECIDE vs 0.260 IVIM). This was expected given that previous work in liver has shown addition of T2 to the IVIM model reduces the value of $f$ [5]. There was a significant $\nu$ fraction in placenta, which is expected, given that it is thought to represent maternal placental perfusion. Fetal blood T2 was lower than the value attributed to adult blood in the literature $(240 \mathrm{~ms})$, however given the dependence of $\mathrm{T} 2$ relaxation time on haematrocrit and saturation, which are known to be different in the fetus, it is a feasible value.

When comparing control and PI myometrial parameters, $f$ was lower in PI compared to control cases. This is expected given the pathophysiology of PI, with minimal spiral artery remodelling, and therefore reduced placental perfusion. 

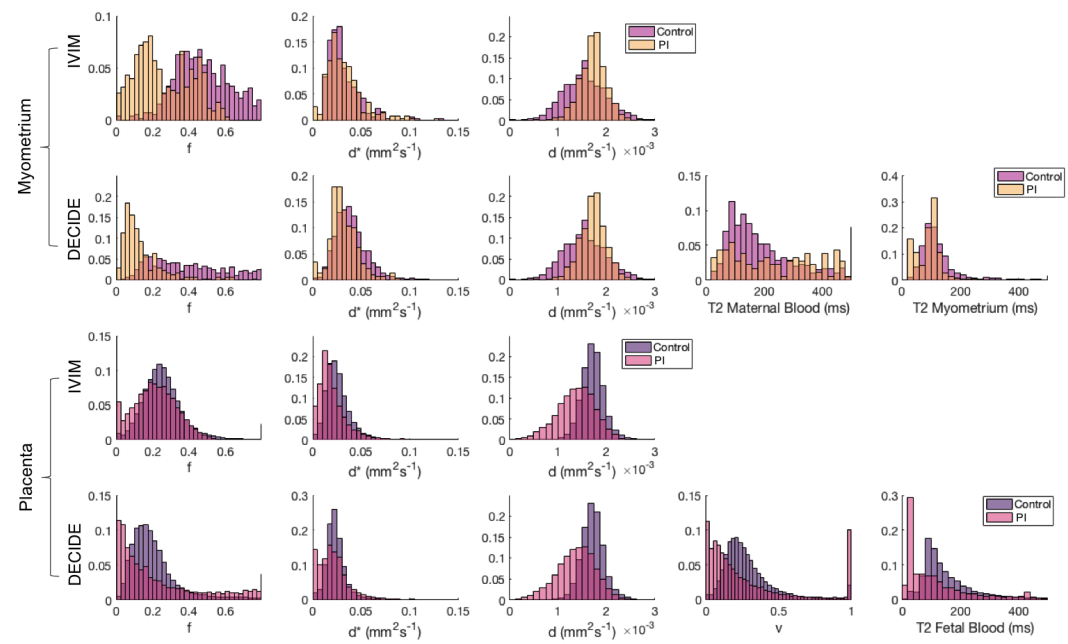

Fig. 4. Combined voxel-wise parameter histograms for the control and PI data. The first two rows show the myometrial data for maternal blood and myometrium, using the IVIM model (row 1) and DECIDE model (row 2). The last two rows show the placenta data using the IVIM model (row 1) and DECIDE model (row 2).

When comparing control and PI placental parameters, $f$ was reduced in PI compared to control placenta with the IVIM model, but not the DECIDE model. This is unexpected, given that histology has shown reduced feto-placental vascular density in PI. We hypothesise this may be due to $f$ measuring flow, rather than volume, which may be increased within the vasoconstricted fetal vasculature. $\nu$ was reduced in PI compared to control cases, in keeping with the myometrial results. The T2 of fetal blood within the placenta is also reduced in PI compared to control cases, which related to a reduced fetal blood saturation in PI compared to control cases. The reduction in fetal blood saturation in PI is reasonable, given the poor placental function. What is particularly interesting is the histogram, showing a peak in PI at $10-20 \%$ saturation. The control data histogram shows little data with a saturation less than $50 \%$. This suggests a greater degree of heterogeneity within the PI placenta, with areas of poorly oxygenated tissue, which may relate to areas of poor maternal perfusion.

In conclusion, we present an extension of the three compartment DECIDE model, with T2 blood and tissue fitting. We applied this to a cohort of control and PI myometrial and placental image data, and showed differences in parameters that may have potential to measure placental pathology. We then presented the use of fetal blood T2 relaxation values for estimating fetal blood saturation non-invasively for the first time in utero. 

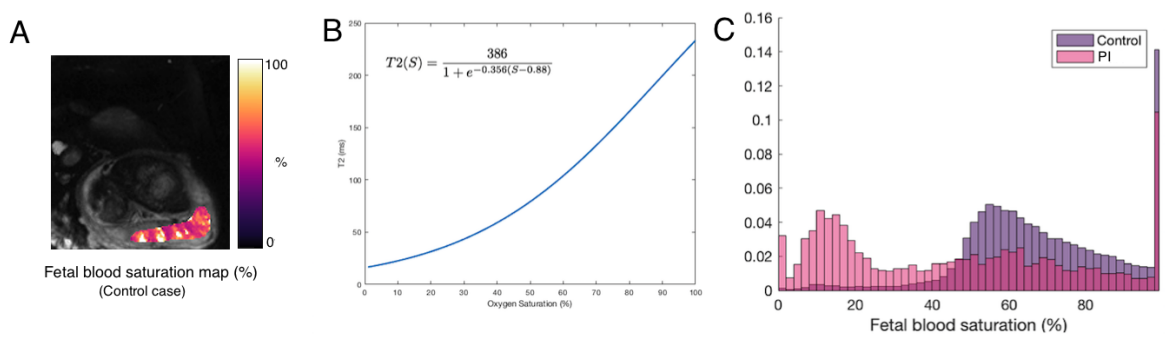

Fig. 5. A) Parametric map of estimated oxygen saturation [7]. B) T2/Sat curve C) Histogram of fetal blood saturation distribution in the placenta.

Acknowledgements We would like to acknowledge the Wellcome Trust (210182/Z/18/Z, $101957 / \mathrm{Z} / 13 / \mathrm{Z}$ ), the National Institute for Health Research (NIHR), the EPSRC (NS/A000027/1) and the Radiological Research Trust.

\section{References}

1. Lawn, J.E., Blencowe, H., Pattinson, R., Cousens, S., Kumar, R., Ibiebele, I., Gardosi, J., Day, L.T., Stanton, C., Committee, L.S.S.S., et al.: Stillbirths: Where? when? why? how to make the data count? The Lancet 377(9775) (2011) 1448-1463

2. Derwig, I., Lythgoe, D.J., Barker, G.J., Poon, L., Gowland, P., Yeung, R., Zelaya, F., Nicolaides, K.: Association of placental perfusion, as assessed by magnetic resonance imaging and uterine artery doppler ultrasound, and its relationship to pregnancy outcome. Placenta 34(10) (Oct 2013) 885-891

3. Derwig, I., Barker, G.J., Poon, L., Zelaya, F., Gowland, P., Lythgoe, D.J., Nicolaides, K.: Association of placental t2 relaxation times and uterine artery doppler ultrasound measures of placental blood flow. Placenta 34(6) (Jun 2013) 474-479

4. Siauve, N., Chalouhi, G.E., Deloison, B., Alison, M., Clement, O., Ville, Y., Salomon, L.J.: Functional imaging of the human placenta with magnetic resonance. Am J Obstet Gynecol 213(4 Suppl) (Oct 2015) S103-S114

5. Jerome, N., d'Arcy, J., Feiweier, T., Koh, D., Leach, M., Collins, D., Orton, M.: Extended t2-ivim model for correction of te dependence of pseudo-diffusion volume fraction in clinical diffusion-weighted magnetic resonance imaging. Physics in medicine and biology 61(24) (2016) N667

6. Melbourne, A., Pratt, R., Owen, D., Sokolska, M., Bainbridge, A., Atkinson, D., Kendall, G., Deprest, J., Vercauteren, T., David, A., et al.: Decide: Diffusionrelaxation combined imaging for detailed placental evaluation, ISMRM (2017)

7. Portnoy, S., Osmond, M., Zhu, M.Y., Seed, M., Sled, J.G., Macgowan, C.K.: Relaxation properties of human umbilical cord blood at 1.5 tesla. Magnetic resonance in medicine $\mathbf{7 7}$ (4) (2017) 1678-1690

8. Siggaard-Andersen, O., Huch, R.: The oxygen status of fetal blood. Acta Anaesthesiologica Scandinavica 39(s107) (1995) 129-135

9. De Bazelaire, C.M., Duhamel, G.D., Rofsky, N.M., Alsop, D.C.: Mr imaging relaxation times of abdominal and pelvic tissues measured in vivo at 3.0 t: preliminary results. Radiology 230(3) (2004) 652-659 